\title{
Continuum contribution to excitonic four-wave mixing due to interaction-induced nonlinearities
}

Birkedal, Dan; Vadim, Lyssenko; Hvam, Jørn Märcher; Sayed, K. EI

Published in:

Physical Review B

Link to article, DOI:

10.1103/PhysRevB.54.R14250

Publication date:

1996

Document Version

Publisher's PDF, also known as Version of record

Link back to DTU Orbit

Citation (APA):

Birkedal, D., Vadim, L., Hvam, J. M., \& Sayed, K. E. (1996). Continuum contribution to excitonic four-wave mixing due to interaction-induced nonlinearities. Physical Review B, 54(20), R14250-R14253.

https://doi.org/10.1103/PhysRevB.54.R14250

\section{General rights}

Copyright and moral rights for the publications made accessible in the public portal are retained by the authors and/or other copyright owners and it is a condition of accessing publications that users recognise and abide by the legal requirements associated with these rights.

- Users may download and print one copy of any publication from the public portal for the purpose of private study or research.

- You may not further distribute the material or use it for any profit-making activity or commercial gain

- You may freely distribute the URL identifying the publication in the public portal 


\title{
Continuum contribution to excitonic four-wave mixing due to interaction-induced nonlinearities
}

\author{
D. Birkedal, V. G. Lyssenko, and J. M. Hvam \\ Mikroelektronik Centret, Technical University of Denmark, Building 345 east, DK-2800 Lyngby, Denmark \\ K. El Sayed \\ Department of Physics, University of Florida, Gainesville, Florida 32611
}

(Received 6 September 1996)

\begin{abstract}
We present an experimental and theoretical investigation of ultrafast transient four-wave mixing of GaAs/ $\mathrm{Al}_{x} \mathrm{Ga}_{1-x} \mathrm{As}$ quantum wells for coherent excitation of exciton and continuum states. The signal appears at the exciton resonance and is shown to consist of two contributions: an intense spectrally narrow part only present for short time delays and a weak exponentially decaying part reflecting the intrinsic coherence properties of the exciton states. The intense part of the signal is due to the excitation of the continuum states mediated by interaction-induced nonlinearities. [S0163-1829(96)50444-3]
\end{abstract}

Recent transient four-wave mixing (TFWM) experiments on semiconductors have shown unexpected results when both discrete exciton states and continuum states have been excited coherently by spectrally broad, ultrafast laser pulses. $^{1-5}$ The decay of the TFWM signal, as a function of delay $\tau$, is significantly faster than the dephasing time of the excitons. However, the width of the spectrally resolved excitonic TFWM response and the real-time evolution of the TFWM signal reflect the intrinsic excitonic dephasing time. The microscopic origin of these observations has so far remained unsettled.

The coherent superposition of a number of eigenstates by ultrafast laser excitation is a well-known concept in atomic physics. In this case, the formation of electron wave packets has been observed in pump-probe experiments on the Rydberg states of, e.g., atomic rubidium. ${ }^{6}$ Similar effects have been investigated in semiconductor physics, where the formation of excitonic wave packets has been observed, either in coupled double quantum wells, ${ }^{7}$ as Bloch oscillations in biased superlattice structures, ${ }^{8}$ or as the pure excitonic eigenstates of a strained quantum well structure. ${ }^{1}$

However, one important difference between atomic and semiconductor wave packets is that the atomic wave packet is unaffected by coherent excitation of the continuum, ${ }^{6,9}$ while the excitonic counterpart is dramatically changed. ${ }^{1,3,4}$ The recovery of the electron wave packet after orbital motion is observed in atomic physics while TFWM experiments on semiconductors indicate a rapid destruction of the excitonic wave packets.

The decay of the strong initial continuum contribution (CC) to the TFWM signal has been attributed to interference between the coherently excited microscopic polarizations rather than dephasing. ${ }^{1}$ However, this explanation implies that coherent excitation of exciton states and continuum states of different quantum wells should show the same CC effect. This aspect has so far not been demonstrated. An alternative explanation is that the CC is due to TFWM with the continuum states. ${ }^{3}$ This TFWM signal would be spectrally centered at and above the continuum edge, which can easily be verified using spectrally resolved TFWM. This explanation also relies on a fast dephasing of the continuum states, which is not consistently demonstrated. TFWM experiments on bulk GaAs for coherent exciton and continuum excitation have indicated values of ${ }^{10} 70 \mathrm{fs}$ and ${ }^{5} 14 \mathrm{fs}$ for the dephasing time of the continuum states, in both cases limited by the laser pulse duration. These values are in clear contradiction to the intrinsic broadening observed in linear transmission experiments, from which dephasing times of several picoseconds can be extracted. Indeed, dephasing times of this order have been reported from experiments with Bloch oscillations using transmissive electro-optic sampling, ${ }^{3}$ a technique that probes directly the intraband polarization of the coherent electron wave packets, and is independent of the interband polarization, contrary to TFWM.

In this paper, we demonstrate experimentally and theoretically that the TFWM signal for coherent excitation of exciton and continuum states is well explained by the interaction induced nonlinearities in the semiconductor Bloch equations (SBE's) with the inclusion of excitation induced dephasing (EID). The TFWM signal at the excitonic resonance is shown to consist of essentially two contributions: a prompt signal due to the coherently excited continuum states mediated by interaction-induced nonlinearities, and a purely excitonic signal, showing the same features as the TFWM signal from a selectively excited excitonic resonance.

These interaction-induced signals are related to the coherent continuum density, in which both dephasing and destructive interference take place. When the continuum is excited with a broad laser pulse, the density gratings associated with each state stay coherent for a time corresponding to the inverse excitation width. For short time delays, the spatial gratings lead to a strong diffraction of the exciton polarization wave. The diffracted excitonic signal reflects then, naturally, the intrinsic features of the exciton, resulting in long realtime decay and hence narrow spectral TFWM lines. For longer time delays, the continuum density has interfered destructively and diffraction in the associated gratings is no longer possible.

The essential new concept of these findings is that the strong initial TFWM signal at an isolated excitonic resonance originates from the excitation of energetically different continuum states. To study these effects in detail, we present 
spectrally resolved TFWM signals to achieve simultaneous real-time and delay-time information. This also allows for a clear separation of different contributions to the TFWM signal.

Excitonic TFWM without coherent continuum excitation has succesfully been modeled with the SBE's, ${ }^{11,12}$ whereas modeling including continuum excitation has been less conclusive. ${ }^{1,5}$ Incoherent scattering processes have in most cases been modeled with a density-independent dephasing time $T_{2}$. However, in semiconductors the dephasing is partially due to Coulomb scattering among the excited particles. In the incoherent regime this simply results in an increased dephasing rate of the induced polarization. ${ }^{13}$ It has been shown recently that the EID may contribute significantly to the excitonic nonlinear response, ${ }^{14}$ and hence to the excitonic TFWM signal. ${ }^{15}$ In fact, it has been shown that on a $\chi^{(3)}$ level the local-field and EID effects contribute to the TFWM signal in a similar way.

A multiple quantum well structure containing ten wells of each of the thicknesses $80 \AA, 100 \AA, 130 \AA$, and $160 \AA$, was grown by molecular-beam expitaxy. The wells are separated by $150-\AA \mathrm{Al}_{0.3} \mathrm{Ga}_{0.7} \mathrm{As}$ barriers, and after the growth the structure was lifted off the substrate and mounted on a sapphire disk. The sample was kept in a liquid He cryostat at 5 $\mathrm{K}$ during the experiments. The linear optical properties of this sample have been reported previously, ${ }^{16}$ showing narrow $(<1 \mathrm{meV})$ exciton absorption peaks and a negligible Stokes shift.

For the TFWM experiments, the sample was excited by parallel linearly polarized 100 -fs pulses from a self-modelocked Ti:sapphire laser. The spectral width of the laser was approximately $15 \mathrm{meV}$, corresponding to near transform limited pulses. The laser spot size was focused to approximately $60 \mu \mathrm{m}$ on the sample surface, and the TFWM experiments were performed in the two-beam self-diffraction geometry, ${ }^{17}$ where two beams with wave vectors $\boldsymbol{k}_{1}$ and $\boldsymbol{k}_{2}$ are incident on the sample under a small angle. The pulse in the $\boldsymbol{k}_{2}$ direction arrives with a delay of $\tau$ after the pulse in the $\boldsymbol{k}_{1}$ direction, and the TFWM signal propagates in the $2 \boldsymbol{k}_{2}-\boldsymbol{k}_{1}$ direction. The signal was dispersed in a $0.6 \mathrm{~m}$ spectrometer and detected by an optical multichannel analyzer with a resulting resolution better than $0.1 \mathrm{meV}$.

The TFWM spectrum is shown as a function of delay in Fig. 1 together with the absorption spectrum of the sample and the laser spectrum. Heavy- and light-hole exciton absorption peaks are seen in the absorption spectrum and the peaks are labeled with their corresponding well thicknesses. The maximum of the laser spectrum is tuned in resonance with the heavy-hole exciton (hhx) of the 100- $\AA$ quantum well. The continuum states of the $160-\AA$ and $130-\AA$ wells are excited for this tuning of the laser, whereas the continuum states of the $100-\AA$ well are marginally excited by the wing of the laser and the hhx of the $80-\AA$ well is excited below resonance. We estimate a carrier density of $\approx 1.5 \times 10^{9} \mathrm{~cm}$

-2 for the resonantly excited $100-\AA$ quantum well.

The decay of the TFWM signal for the hhx of the 80 - $\AA$ well is long and it has a delayed maximum since the laser predominantly excites the localized tail of the exciton resonance. ${ }^{4}$ The TFWM signal is in this case due to a photon echo involving destructive interference and rephasing of the polarizations of the localized states. The TFWM intensity for

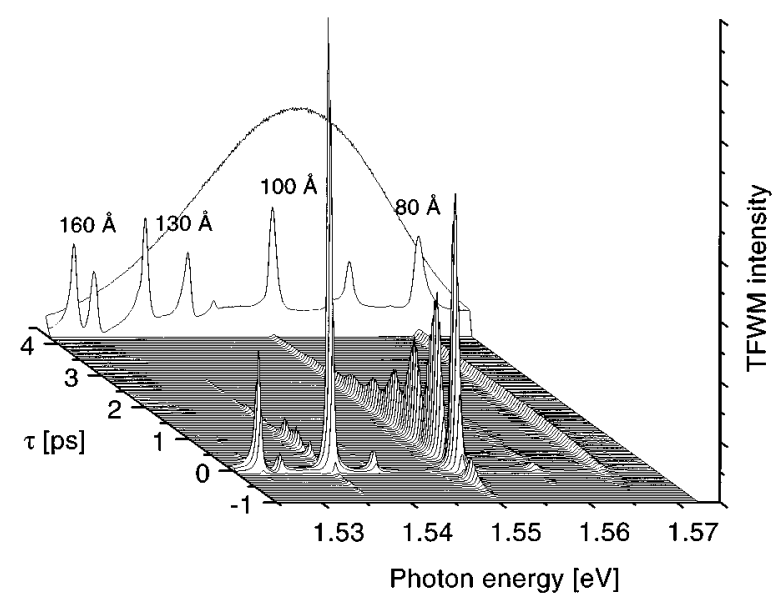

FIG. 1. Transient four-wave mixing spectra as a function of delay. The absorption profile and the laser spectrum are also shown.

the hhx resonance of the $100-\AA$ well shows pronounced heavy-hole-light-hole quantum beats due to coherent excitation of heavy- and light-hole excitons.

The TFWM signals from the hhx of the 130- $\AA$ and $160-\AA$ wells show a dramatic change as compared to those from the $100-\AA$ and $80-\AA$ wells. In these cases where the continuum states are excited coherently with the excitonic states we observe the aforementioned CC to the TFWM. A beat structure is seen in the TFWM signal from the hhx of the $130-\AA$ well due to beats with the light-hole exciton and the $2 s$ state of the hhx, which is well resolved in the absorption spectrum. However, it is evident from the data that these beats are of much lower intensity than the CC to the TFWM signal. In fact, the intensity of the TFWM signal from the hhx of $130-\AA$ well should be compared to that of the hhx of the $80-\AA$ well since these have comparable excitation strengths. The hhx of the $160-\AA$ quantum well is even more weakly excited and still shows a quite intense TFWM signal for short delay times.

It is shown in Fig. 1 how the excitonic TFWM signal is dramatically changed depending on whether the continuum states are excited or not. However, it is clearly demonstrated in this experiment that this effect is not related to incoherent scattering processes, since these will lead to spectral broadening or faster real-time decay. We observe no additional broadening of the hhx resonances in the TFWM spectra for continuum excitation. On the contrary, we observe an increasing spectral narrowing compared to the linewidths of the absorption spectrum for increasing continuum excitation.

It is also shown that the $\mathrm{CC}$ phenomena only occur when the exciton and the continuum belong to the same quantum wells. When the laser is tuned in resonance with the hhx of the 100- $\AA$ quantum well, the continuum states of the hhx of the 130- $\AA$ quantum well are also excited and no CC effect is observed in the TFWM signal from the 100- $\AA$ hhx resonance. This proves that the $\mathrm{CC}$ is not related to simple (photon-echo-type) interferences in the polarizations, as earlier proposed, ${ }^{1}$ but rather due to interaction-induced nonlinearities.

For a direct comparison, we show the TFWM intensities at the hhx resonances of the $100-\AA, 130-\AA$, and $160-\AA$ quantum wells in Fig. 2. The curves are normalized and displaced 


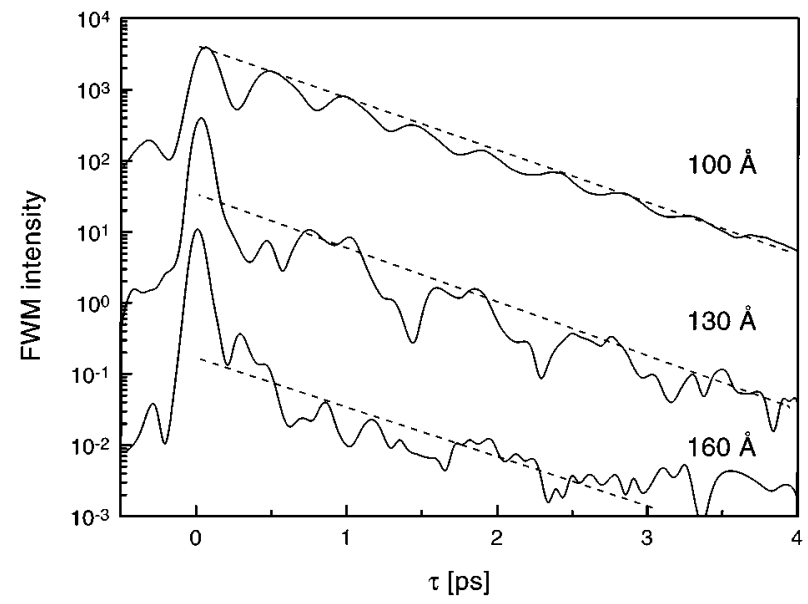

FIG. 2. Four-wave mixing intensities at the heavy-hole exciton resonance of the $100-\AA, 130-\AA$, and $160-\AA$ quantum wells for the excitation shown in Fig. 1.

for clarity. It is seen how the CC decays faster for increasing continuum excitation. It is also seen that the initial $\mathrm{CC}$ increases in intensity for increasing continuum excitation as compared with the exponential excitonic part.

To confirm these experimental findings we present numerical simulations based on the SBE's, which are the equations of motion of the occupation numbers $f_{j \mathbf{k}}=\left\langle a_{j \mathbf{k}}^{\dagger} a_{j \mathbf{k}}\right\rangle$ and the microscopic polarizations $P_{\mathbf{k}}=\left\langle a_{h \mathbf{k}}^{\dagger} a_{e \mathbf{k}}\right\rangle$. For a simple two-band model, the particle index $j$ is $e$ for the electrons and $h$ for the holes. The SBE read

$$
\begin{gathered}
\frac{\partial}{\partial t} P_{k}=-i\left[e_{e k}(t)+e_{h k}(t)+i \gamma(t)\right] P_{k}(t) \\
+i\left[1-f_{e k}(t)-f_{h k}(t)\right] \Omega_{k}^{R}(t), \\
\frac{\partial}{\partial t} f_{j k}=-2 \operatorname{Im}\left[\Omega_{k}^{R}(t) P_{k}^{*}(t)\right] .
\end{gathered}
$$

The mean-field, or Hartree-Fock part of the carrier-carrier interaction contributes to the Rabi frequency $\Omega^{R}$ (local-field) and to the single-particle energies $\hbar e_{i}$ (band-gap renormalization):

$$
\begin{gathered}
\hbar \Omega_{k}^{R}(t)=\frac{d_{k} E(t)}{2}+\sum_{\mathbf{q}} V_{q} P_{|\mathbf{k}-\mathbf{q}|}(t), \\
\hbar e_{j k}(t)=\varepsilon_{j k}-\sum_{\mathbf{q}} V_{q} f_{j|\mathbf{k}-\mathbf{q}|}(t) .
\end{gathered}
$$

Within the rotating wave approximation, $E(t)$ denotes only the pulse envelope. $d_{k}$ is the interband optical matrix element. $V_{q}=2 \pi e^{2} / \epsilon_{0} q$ is the bare 2D Coulomb potential with the background dielectric constant $\epsilon_{0}$. The single-particle energies are given by $\varepsilon_{e k}=(\hbar k)^{2} / 2 m_{e}-\Delta_{0}$, and $\varepsilon_{h k}=(\hbar k)^{2} / 2 m_{h}$. Here, $\Delta_{0}=\hbar \omega_{0}-E_{g}$ is the detuning between the central frequency $\omega_{0}$ of the pulse and the unrenormalized band gap $E_{g}$. We include in the simulation a phenomenological, carrier-independent dephasing rate $\left(\gamma_{0}=1 / T_{2}\right)$, as well as an excitation-induced part (see Refs. 14,15 , and 18):

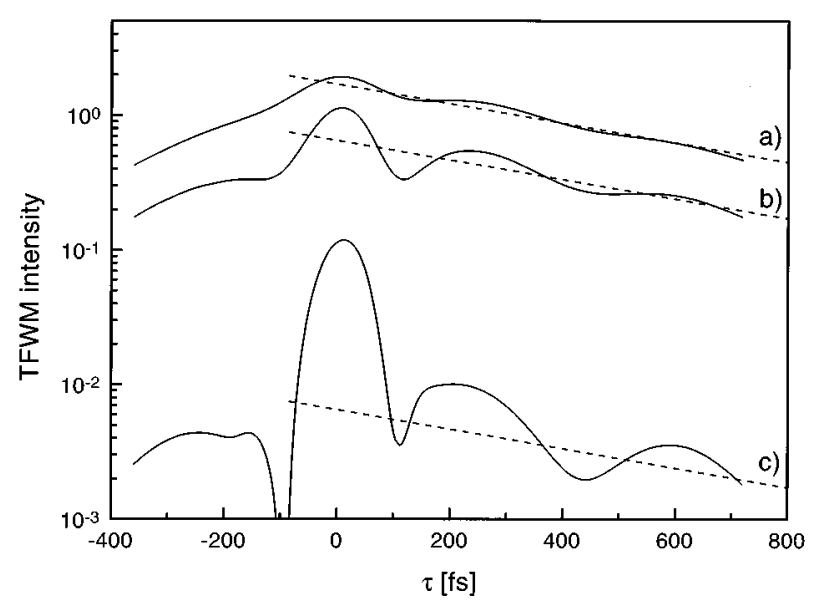

FIG. 3. Four-wave mixing intensities at the $1 s$ exciton resonance simulated from the SBE with EID for detunings from the $1 s$ exciton of (a) $0 \mathrm{meV}$, (b) $10.5 \mathrm{meV}$, and (c) $23 \mathrm{meV}$. The dashed lines show an exponential decay with $T_{2}=750 \mathrm{fs}$.

$$
\gamma(t)=\gamma_{0}+\gamma^{\prime} \frac{1}{2} \sum_{k}\left[f_{e k}(t)+f_{h k}(t)\right] .
$$

The main difference between the EID and the HartreeFock nonlinearities is the number of states they involve. The Coulomb potential is localized in momentum space and couples only a limited number of states. The EID, on the contrary, models the decay of the polarization due to scatterings with other particles. This interaction is not local, because an exciton can be scattered by essentially any free carrier or other excitons. With the inclusion of Eq. (3) in Eq. (1) we find that the polarization wave associated with an exciton transition can scatter in the density gratings formed by the continuum states through the scattering potential $\gamma^{\prime}$, thus giving rise to an additonal four-wave mixing signal at the exciton transition energy. ${ }^{19}$

The SBE are solved for a TFWM geometry ${ }^{20}$ using the numerical procedure proposed in Ref. 21 with and without EID. The exciting laser field $E(t)$ is taken to be the sum of two delayed, but otherwise identical, Gaussians with an intensity FWHM of 72 fs. Figure 3 shows the resulting TFWM signals computed with EID at the spectral possition of the $1 \mathrm{~s}$ exciton for excitations with a detuning from the $1 \mathrm{~s}$ exciton of (a) 0 , (b) $10.5 \mathrm{meV}$, and (c) $23 \mathrm{meV}$. In Fig. 3, $\gamma_{0}$ is taken to be $1 / 1200 \mathrm{fs}$ and $\gamma^{\prime}=3 \times 10^{-13} \mathrm{~cm}^{2} / \mathrm{fs}$. The latter value corresponds to the experimentally determined value for EID due to nonthermalized carriers, ${ }^{13}$ and to the value reported in Ref. 12. For the calculations without EID we take $\gamma_{0}=1 / 750 \mathrm{fs}$, ensuring the same average dephasing time. We find that for cases (a) and (b) the simulations with and without EID give the same spectral width of the TFWM signal, whereas in case (c) we find a slight line narrowing if EID is included, consistent with the experiments. ${ }^{19}$

The simulated data show $1 s-2 s$ beats and a clear CC to the signal in (b) and (c). In Fig. 3(c) the CC to the signal has about equal contributions from local field effects and EID, the latter of which is responsible for the temporal narrowing. The calculated intensity ratio between the initial peak and the exponential tail is smaller than experimentally observed. 
However, a better agreement with the experiment can only be achieved by increasing the EID coupling strength $\gamma^{\prime}$.

These observations show that the excitonic TFWM signal as a function of delay is composed by two contributions for coherent excitation of exciton and continuum states: A strong initial CC that is due to coherent excitation of the continuum states mediated through interaction-induced nonlinearities, and a weaker exponentially decaying part, nearly identical to that of the resonantly excited excitons, independent of the continuum excitation.

It is also shown that, in the case of coherent excitation of exciton and continuum states, the decay of the excitonic TFWM is not directly related to the dephasing time of the continuum states, but rather to the width of the excitation.
Hence, pulse limited decay times obtained in this type of experiments $^{10,5}$ should not be taken as an indication of extremely fast dephasing times for the continuum interband polarization. Instead, much longer dephasing times should be considered, as obtained with, e.g., transmissive electro-optic sampling techniques, ${ }^{3}$ in agreement with linear transmission data.

We would like to thank C.B. Sørensen, III-V NANOLAB, for the sample preparation. This work was supported by the Center for Nanostructures (CNAST) and the Danish Natural Science Research Council. K. El Sayed acknowledges support from the National Science Foundation through Grant No. DMR-9520191.
${ }^{1}$ J. Feldmann, T. Meirer, G. von Plessen, M. Koch, E. Göbel, P. Thomas, G. Bacher, C. Hartmann, H. Schweizer, W. Schäfer, and H. Nickel, Phys. Rev. Lett. 70, 3027 (1993).

${ }^{2}$ U. Siegner, M.-A. Mycek, S. Glutsch, and D. S. Chemla, Phys. Rev. Lett. 74, 470 (1995).

${ }^{3}$ P. Leisching, T. Dekorsy, H. J. Bakker, H. Kurz, and K. Köhler, Phys. Rev. B 51, 18015 (1995).

${ }^{4}$ J. Erland, D. Birkedal, V. G. Lyssenko, and J. M. Hvam, J. Opt. Soc. Am. B 13, 981 (1996).

${ }^{5}$ M. U. Wehner, D. Steinbacher, M. Wegener, T. Marschner, and W. Stolz, J. Opt. Soc. Am. B 13, 977 (1996).

${ }^{6}$ A. ten Wolde, L. D. Noordam, A. Lagendijk, and H. B. van Linden van den Heuvell, Phys. Rev. Lett. 61, 2099 (1988).

${ }^{7}$ K. Leo, J. Shah, E. O. Göbel, T. C. Damen, S. Schmitt-Rink, W. Schäfer, and K. Köhler, Phys. Rev. Lett. 66, 201 (1991).

${ }^{8}$ K. Leo, P. H. Bolivar, F. Brüggermann, R. Schwedler, and K. Köhler, Solid State Commun. 84, 943 (1992).

${ }^{9}$ H. B. van Linden van den Heuvell (private communications).

${ }^{10}$ A. Lohner, K. Rick, P. Leisching, A. Leitenstorfer, T. Elsasser, T. Kuhn, F. Rossi, and W. Stoltz, Phys. Rev. Lett. 71, 77 (1993).

${ }^{11}$ S. Schmitt-Rink and D. S. Chemla, Phys. Rev. Lett. 57, 2752 (1986); M. Lindberg and S. W. Koch, Phys. Rev. B 38, 3342 (1988); K. Leo, M. Wegener, J. Shah, D. S. Chemla, E. O. Göbel, T. C. Damen, S. Schmitt-Rink, and W. Schäefer, Phys. Rev. Lett. 65, 1340 (1990); M. Wegener, D. S. Chemla, S. Schmitt-Rink, and W. Schäefer, Phys. Rev. A 42, 5675 (1990);
S. Weiss, M.-A. Mycek, J.-Y. Bigot, S. Schmitt-Rink, and D. S. Chemla, Phys. Rev. Lett. 69, 2685 (1992); H. Haug and S. W. Koch, Quantum Theory of the Optical and Electronic Properties of Semiconductors, 2nd ed. (World Scientific, Singapore, 1993).

${ }^{12}$ D.-S. Kim, J. Shah, J. E. Cunningham, T. C. Damen, W. Schäefer, and M. Hartmann, Phys. Rev. Lett. 68, 1006 (1992).

${ }^{13}$ L. Schultheis, J. Kuhl, A. Honold, and C. W. Tu, Phys. Rev. Lett. 57, 1635 (1986); A. Honold, L. Schultheis, J. Kuhl, and C. W. Tu, Phys. Rev. B 40, 6442 (1989).

${ }^{14}$ H. Wang, K. B. Ferrio, D. G. Steel, Y. Z. Hu, R. Binder, and S. W. Koch, Phys. Rev. Lett. 71, 1261 (1993).

${ }^{15}$ H. Wang, K. B. Ferrio, D. G. Steel, P. R. Berman, Y. Z. Hu, R. Binder, and S. W. Koch, Phys. Rev. A 49, R1551 (1994).

${ }^{16}$ D. Birkedal, J. Singh, V. G. Lyssenko, J. Erland, and J. M. Hvam, Phys. Rev. Lett. 76, 672 (1996).

${ }^{17}$ T. Yajima and Y. Taira, J. Phys. Soc. Jpn. 47, 1620 (1979).

${ }^{18}$ Y. Z. Hu, R. Binder, S. W. Koch, S. T. Cundiff, H. Wang, and D. G. Steel, Phys. Rev. B 49, 14382 (1994).

${ }^{19}$ K. El Sayed, D. Birkedal, V. G. Lyssenko, and J. M. Hvam (unpublished).

${ }^{20}$ M. Lindberg, R. Binder, and S. W. Koch, Phys. Rev. A 45, 1865 (1992); T. Rappen, U. Peter, M. Wegener, and W. Schäefer, Phys. Rev. B 48, 4879 (1993).

${ }^{21}$ L. Bányai, D. B. Tran Thoai, E. Reitsamer, H. Haug, D. Steinbach, M. U. Wehner, M. Wegener, T. Marschner, and W. Stoltz, Phys. Rev. Lett. 75, 2188 (1995). 